\title{
Update on nCTEQ PDFs: nuclear PDF uncertainties and LHC applications
}

\author{
A. Kusina ${ }^{* a}$, K. Kovařík ${ }^{b}$, T. Ježo ${ }^{c}$, D. B. Clark ${ }^{a}$, F. I. Olness ${ }^{a}$, I. Schienbein ${ }^{d}$, J. Y. Yu ${ }^{a}$ \\ ${ }^{a}$ Southern Methodist University, Dallas, TX 75275, USA \\ ${ }^{b}$ Institut für Theoretische Physik, Westfälische Wilhelms-Universität Münster, \\ Wilhelm-Klemm-Straße 9, D-48149 Münster, Germany \\ ${ }^{c}$ Department of Physics, University of Durham, Durham DH1 3LE, UK \\ Department of Mathematical Sciences, University of Liverpool, Liverpool L69 3BX, UK \\ ${ }^{d}$ Laboratoire de Physique Subatomique et de Cosmologie, Université Grenoble-Alpes, \\ CNRS/IN2P3, 53 avenue des Martyrs, 38026 Grenoble, France \\ E-mail: akusina@smu.edu, karol.kovarikeuni-muenster.de, \\ T.Jezo@liverpool.ac.uk, dbclarkesmu.edu, olness@smu.edu, \\ ingo.schienbein@lpsc.in2p3.fr, yu@physics.smu.edu
}

\begin{abstract}
We present updated nCTEQ nuclear parton distribution functions with errors including pion production data from RHIC. We compare them with the results of other groups and present selected LHC applications.
\end{abstract}

XXII. International Workshop on Deep-Inelastic Scattering and Related Subjects,

28 April - 2 May 2014

Warsaw, Poland

${ }^{*}$ Speaker. 


\section{Introduction}

Nuclear parton distribution functions (PDFs) are an important ingredient required for the description of heavy ion collisions done at the LHC and RHIC. They are also important for the precise determination of the free-proton PDFs, as some of the data used in the free-proton fits are obtained using nuclear targets, e.g. neutrino DIS experiments. In this contribution we report on the updated analysis of the $\mathrm{NCTEQ}$ nuclear parton distributions and mention some of its application to LHC physics.

In the global analysis presented below we are using the same framework (parametrization, etc.) as in the previous $\mathrm{nCTEQ}$ fits $[1,2,3,4]$ with the addition of an error analysis. The free-proton baseline PDFs [5] are based on the CTEQ6.1 proton fit [6]. For the error estimate we use the Hessian method that was introduced in refs. [7, 8]. This framework was already briefly discussed in the DIS2013 proceedings [9] and we refer the reader to that text and to refs. [2, 3] for more details about it.

The new ingredient presented in the current paper is the addition of the single pion production data from RHIC [10, 11]. The results shown below are still preliminary; the official release of the nCTEQ PDFs will be available later this year.

\section{Global analysis}

The bulk of the data used in the current analysis is DIS and DY data on different nuclear targets, ${ }^{1}$ which after kinematical cuts gives 708 data points. Additionally we include 32 data points of $\pi^{0}$ production at RHIC $[10,11] .^{2}$ All of these data are included with weight of one in the global $\chi^{2}$ function.

We present here two fits: (i) excluding the pion data, and (ii) including the pion data. For both of them we used 16 free fitting parameters, and in both cases it allowed us to obtain very good fits with $\chi^{2} /$ dof respectively equal to 0.87 (without pion) and 0.85 (with pion). In figure 1 we compare the two fits. We show there nuclear correction factors (left panel) and PDFs themselves (right panel) in case of lead at a scale of $Q=10 \mathrm{GeV}$. We can see that, as expected, the biggest impact of the pion data is seen in case of the gluon PDF where there is a shape change. Additionally, if we compare gluon PDF of the above fits with the gluon from the EPS09 fit [12] (where pion data [10] was included with weight of 20) we will see that the gluon of the fit with pion data is closer to the EPS09 one.

In figure 2 we compare the $\mathrm{NCTEQ}$ fit including pion data with fits from other groups providing nuclear PDFs [12, 13, 14]. We can see a good agreement between the $\mathrm{nCTEQ}$ and both EPS09 [12] and DSSZ [13] PDFs in case of gluon and sea quarks. However there is a big difference in case of the valence distributions, especially for $d$-valence. This difference is well understood, and it is related to the assumptions for the parametrization used during the fit. In both the EPS09 and DSSZ approaches the nuclear correction for $u$-valence and $d$-valence PDFs is universal which means that

\footnotetext{
${ }^{1}$ For details on which data sets are used see Tables I, II and III in ref. [2]. Note also that we do not include here any of the neutrino DIS data.

${ }^{2}$ We used the following kinematical cuts: for DIS and DY data $Q>2 \mathrm{GeV}$ and $W>3.5 \mathrm{GeV}$, and for the single pion production $p_{T} \in(1.7,15.5) \mathrm{GeV}$.
} 

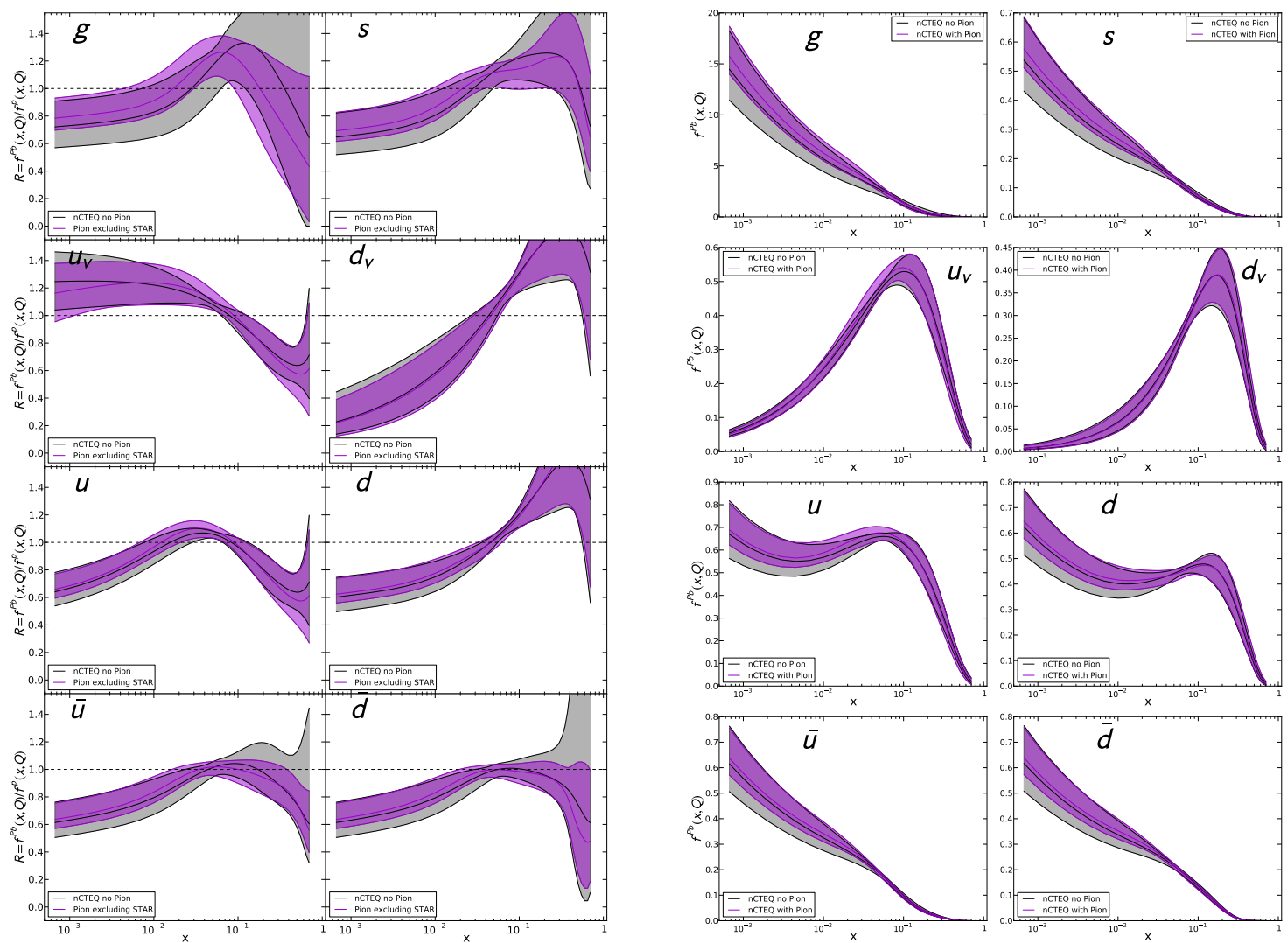

Figure 1: Comparison of $\mathrm{nCTEQ}$ fits with (violet) and without (gray) pion data. On the left we show nuclear modification factors defined as ratios of proton PDFs bound in lead to the corresponding free proton PDFs, and on the right we show the actual bound proton PDFs for lead. In both cases scale is equal to $Q=10 \mathrm{GeV}$.

the two are tied together, whereas in the $\mathrm{NCTEQ}$ framework we treat them as independent (having separate fitting parameters). If we look at the valence distributions obtained by the HKN [14] group, where $u$-valence and $d$-valence are also treated as independent, we can see that the nCTEQ distributions lie in between those of HKN and EPS09 or DSSZ. This behavior clearly illustrates the fact that there is not enough data to properly constrain the nuclear PDFs, and shows that error PDFs obtained by each of the groups do not fully quantify the actual uncertainties related with the nuclear PDFs. Errors obtained in our analysis are bigger than in PDFs from other groups, which can be seen as a more conservative representation of the uncertainties. However, for a most realistic estimate of the actual PDF-related uncertainties we should combine errors obtained by different groups.

\section{Comparison with data and some LHC predictions}

Here we show how well our fit describes the fitted data. As an example, in figure 3a, we show data for the ratio of $F_{2}$ structure functions [15, 16, 17] for iron and deuteron overlaid with our predictions (violet band) and with central predictions of EPS09 and HKN07 fits. We can see that 

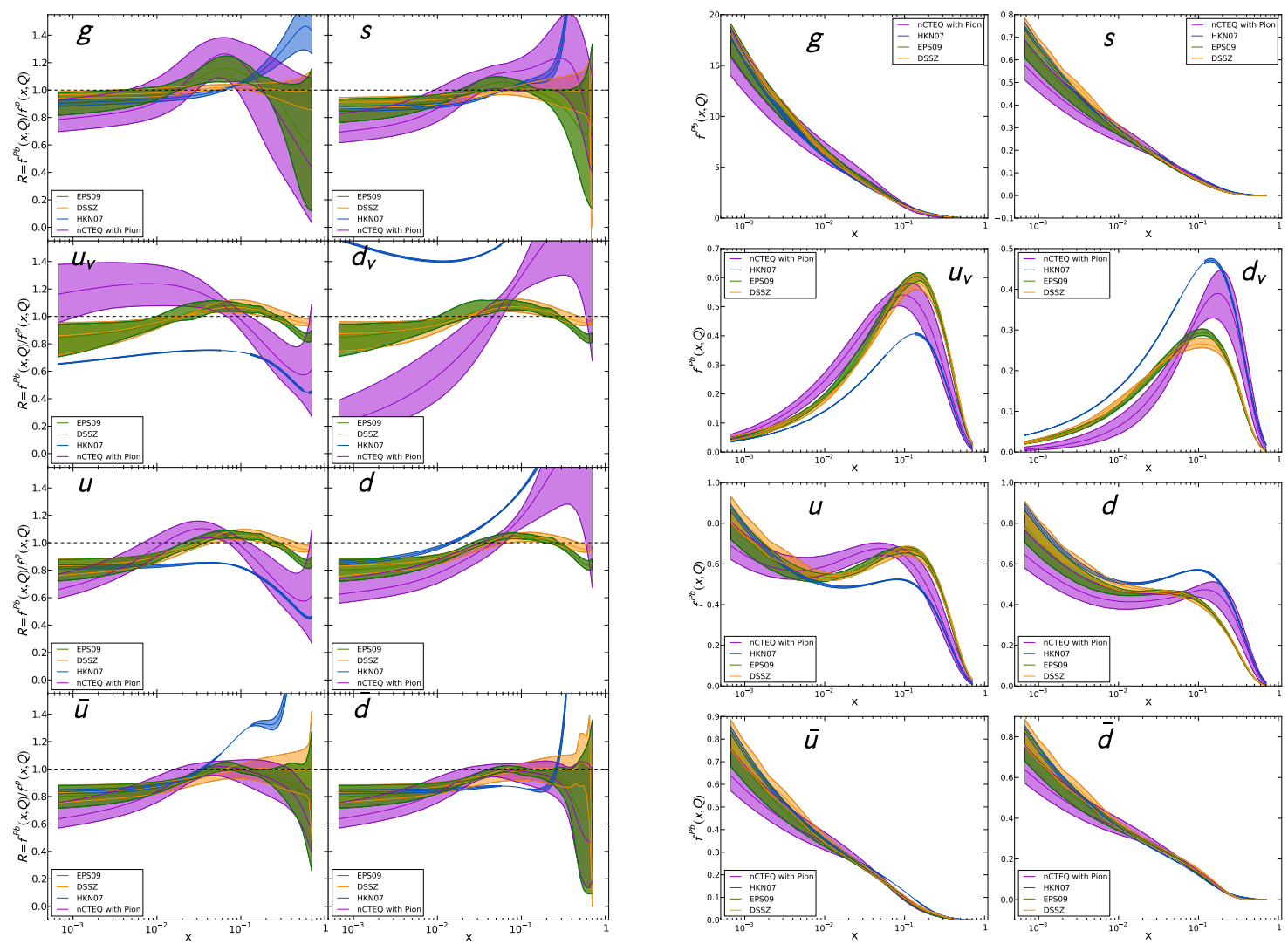

Figure 2: Comparison of $\mathrm{nCTEQ}$ "pion fit" (violet) with results from other groups: EPS09 [12] (green), DSSZ [13] (orange), HKN07 [14] (blue). The left panel shows nuclear modification factors for lead and the right panel the actual PDFs of a proton bound in lead, both at scale of $10 \mathrm{GeV}$.

our PDFs provide a very good description of the data, also the error bands quite precisely reflect the errors of the data.

In figure $3 b$ we look at the pion data that was used during our analysis. In this figure we show the nuclear modification of the pion yield:

$$
R_{\mathrm{dAu}}^{\pi}=\frac{\frac{1}{2 A} d^{2} \sigma_{\pi}^{\mathrm{dAu}} / d p_{T} d y}{d^{2} \sigma_{\pi}^{\mathrm{pp}} / d p_{T} d y}
$$

measured in RHIC and compare it with predictions computed using our fit and using the EPS09 fit (which also used some of these data). We can see that the experimental errors are very large, especially in case of the STAR data, and this is reflected in our error band (violet). Comparing with EPS09 we can see that our central values are close but EPS09 features much smaller uncertainties. This is caused by the fact that in EPS09 analysis only the PHENIX data was used (with much lower experimental errors) and because the PHENIX data was included with weight of 20, magnifying its impact, whereas we used a weight of 1.

As a last thing we present predictions for $W^{+}$production in lead collisions at the LHC calculated at NLO using our new fit. ${ }^{3}$ In a recent ATLAS analysis [19] the rapidity distributions of

\footnotetext{
${ }^{3}$ For calculating NLO distributions for $W^{+}$and $\mu^{+}$the FEWZ code [18] was used.
} 


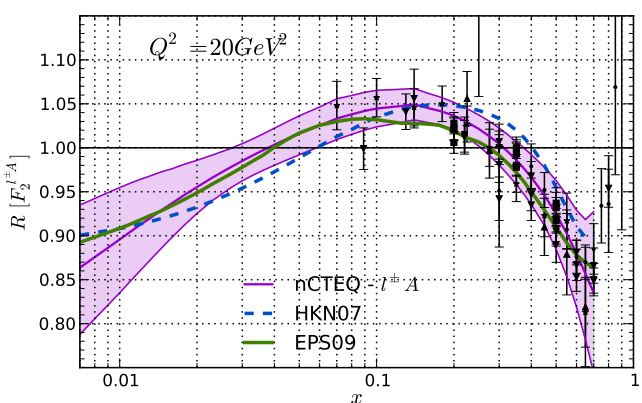

(a)

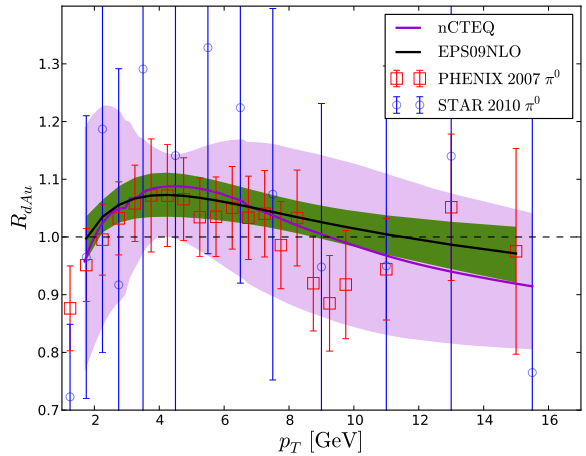

(b)

Figure 3: (a) Ratio of $F_{2}$ structure functions for iron and deuteron calculated with $\mathrm{nCTEQ}$ "pion fit" overlaid with fitted data and results from EPS09 and HKN07; (b) $R_{\mathrm{dAu}}^{\pi}$ computed with nCTEQ and EPS09 [12] PDFs overlaid with PHENIX [10] and STAR [11] data.
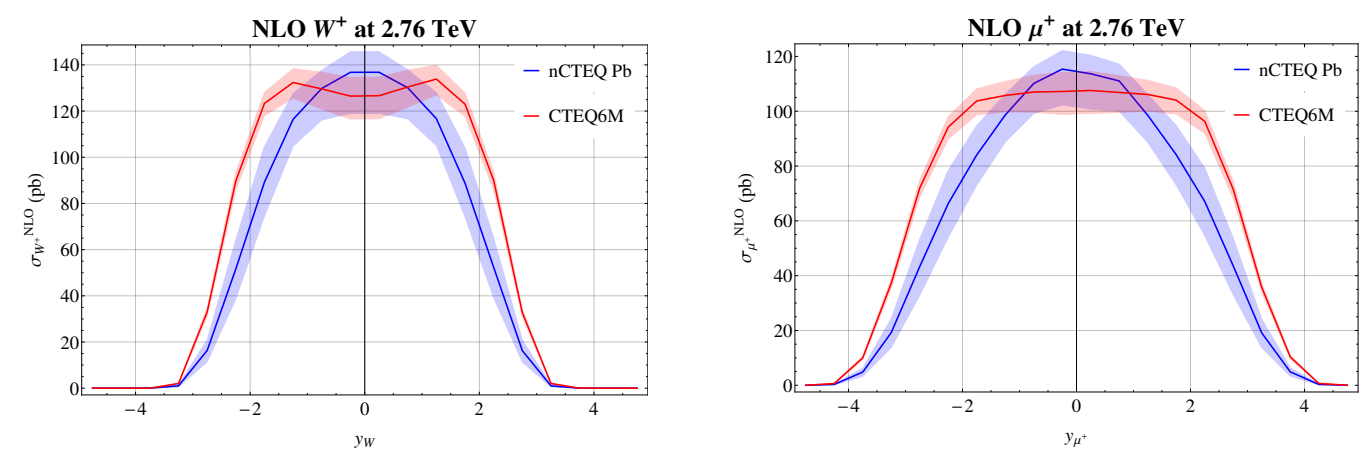

Figure 4: Comparison of NLO $W^{+}$(left) and $\mu^{+}$(right) rapidity distributions in case of proton (red) and lead (blue) collisions with $\sqrt{s}=2.76 \mathrm{TeV}$.

the muon originating from the decay of $W^{+}$boson was measured. In this analysis free-proton PDFs were used to estimate a theoretical prediction for this distribution, which was advocated to be sufficient due to the substantial experimental errors and limited knowledge of the nuclear PDFs. However, as can be seen in figure 4 distributions obtained with the free-proton PDFs (red) and with the nuclear PDFs (blue) are very different (even for $\mu^{+}$), and we should become sensitive to these differences with more data.

\section{Conclusion}

We have presented a preliminary analysis of $\mathrm{nCTEQ}$ nuclear PDFs introducing an error analysis to our framework, and including pion production data that we have not used before.

We performed a comparison with the results of other groups [12,13,14] and found our PDFs to be in reasonably good agreement with them. Also the discrepancies occurring in the valence distributions are well understood. The estimates of errors provided by our fit are larger than those 
of other groups. The possible reason for this is related to the number of free parameters used in the fit and with the flexibility of the parametrization itself.

The presented results are still preliminary. The official $\mathrm{nCTEQ}$ release (providing PDF grids) will appear later this year.

\section{Acknowledgments}

This work was partially supported by the U.S. Department of Energy under grant DE-FG0213ER41996, and the Lighter Sams Foundation.

\section{References}

[1] I. Schienbein, et al., Phys.Rev. D77 (2008) 054013, 0710.4897.

[2] I. Schienbein, et al., Phys.Rev. D80 (2009) 094004, 0907.2357.

[3] K. Kovarik, et al., Phys.Rev.Lett. 106 (2011) 122301, 1012.0286.

[4] T. Stavreva, et al., JHEP 1101 (2011) 152, 1012.1178.

[5] J. Owens, et al., Phys.Rev. D75 (2007) 054030, hep-ph/ 0702159.

[6] D. Stump, et al., JHEP 0310 (2003) 046, hep-ph / 0303013.

[7] J. Pumplin, D. Stump, and W. Tung, Phys.Rev. D65 (2001) 014011, hep-ph / 0008191.

[8] J. Pumplin, et al., Phys.Rev. D65 (2001) 014013, hep-ph/ 0101032.

[9] K. Kovarik, et al., PoS DIS2013 (2013) 274, 1307 . 3454.

[10] PHENIX Collaboration Collaboration, S. Adler et al., Phys.Rev.Lett. 98 (2007) 172302, nucl-ex/0610036.

[11] STAR Collaboration Collaboration, B. Abelev et al., Phys.Rev. C81 (2010) 064904, 0912.3838.

[12] K. Eskola, H. Paukkunen, and C. Salgado, JHEP 0904 (2009) 065, 0902.4154.

[13] D. de Florian, R. Sassot, P. Zurita, and M. Stratmann, Phys.Rev. D85 (2012) 074028, 1112 . 6324.

[14] M. Hirai, S. Kumano, and T.-H. Nagai, Phys.Rev. C76 (2007) 065207, 0709 . 3038.

[15] A. Bodek et al., Phys. Rev. Lett. 50 (1983) 1431.

[16] J. Gomez et al., Phys. Rev. D49 (1994) 4348-4372.

[17] S. Dasu et al., Phys. Rev. D49 (1994) 5641-5670.

[18] R. Gavin, Y. Li, F. Petriello, and S. Quackenbush, Comput.Phys.Commun. 182 (2011) 2388-2403, 1011.3540.

[19] "Measurement of the $W \rightarrow \mu v$ charge asymmetry and centrality dependence in $\mathrm{Pb}+\mathrm{Pb}$ collisions at $\sqrt{s_{N N}}=2.76 \mathrm{TeV}$ with the ATLAS detector", preprint ATLAS-CONF-2013-106 CERN, Geneva (Nov, 2013). 